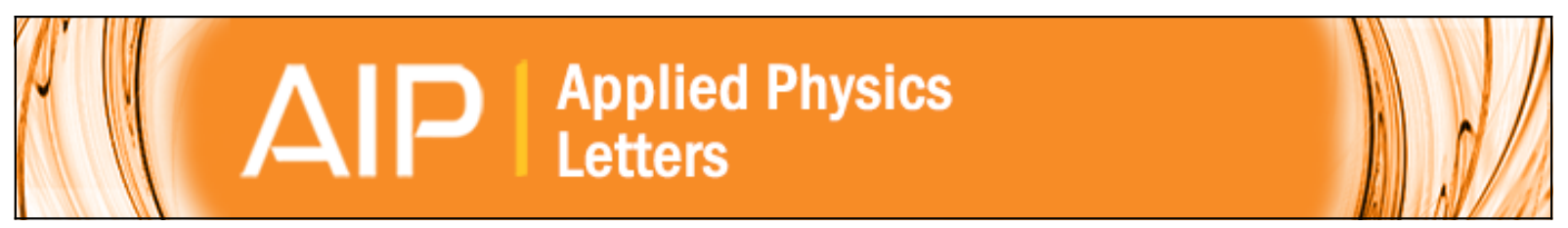

\title{
Atomic layer epitaxy of Ruddlesden-Popper SrO(SrTiO3)n films by means of metalorganic aerosol deposition
}

M. Jungbauer, S. Hühn, R. Egoavil, H. Tan, J. Verbeeck, G. Van Tendeloo, and V. Moshnyaga

Citation: Applied Physics Letters 105, 251603 (2014); doi: 10.1063/1.4905055

View online: http://dx.doi.org/10.1063/1.4905055

View Table of Contents: http://scitation.aip.org/content/aip/journal/apl/105/25?ver=pdfcov

Published by the AIP Publishing

\section{Articles you may be interested in}

Epitaxial strontium titanate films grown by atomic layer deposition on $\mathrm{SrTiO3-buffered} \mathrm{Si}(001)$ substrates J. Vac. Sci. Technol. A 31, 01A136 (2013); 10.1116/1.4770291

Strain relaxation of epitaxial ( $\mathrm{Ba} 0.6 \mathrm{Sr} 0.4$ ) ( Zr 0.3 Ti 0.7 ) O 3 thin films grown on SrTiO 3 substrates by pulsed laser deposition

J. Appl. Phys. 107, 106101 (2010); 10.1063/1.3380528

Microstructure of compositionally-graded ( $\mathrm{Ba} 1-\mathrm{x} \mathrm{Sr} x$ ) Ti O 3 thin films epitaxially grown on La $0.5 \mathrm{Sr} 0.5$ Co O 3 -covered (100) La Al O 3 substrates by pulsed laser deposition

J. Appl. Phys. 97, 093503 (2005); 10.1063/1.1882766

Epitaxial growth of the first five members of the $\mathrm{Sr} n+1 \mathrm{Ti} n$ O $3 n+1$ Ruddlesden-Popper homologous series Appl. Phys. Lett. 78, 3292 (2001); 10.1063/1.1371788

Effects of precursors and substrate materials on microstructure, dielectric properties, and step coverage of (Ba, Sr)TiO 3 films grown by metalorganic chemical vapor deposition

J. Appl. Phys. 87, 124 (2000); 10.1063/1.371833

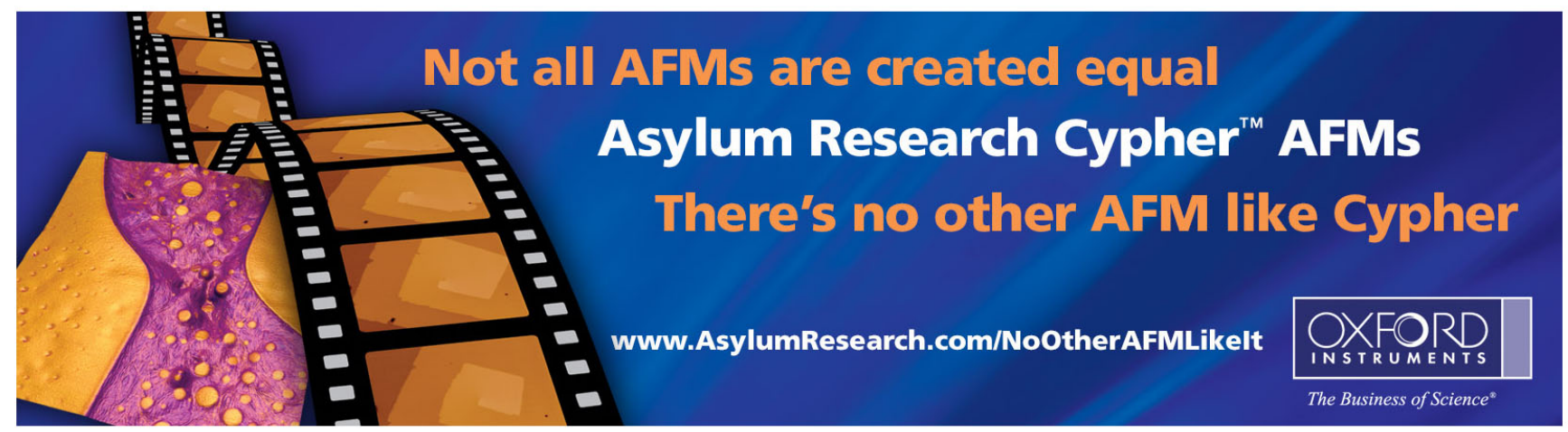




\title{
Atomic layer epitaxy of Ruddlesden-Popper $\mathrm{SrO}\left(\mathrm{SrTiO}_{3}\right)_{\mathrm{n}}$ films by means of metalorganic aerosol deposition
}

\author{
M. Jungbauer, ${ }^{1}$ S. Hühn, ${ }^{1}$ R. Egoavil, ${ }^{2}$ H. Tan, ${ }^{2}$ J. Verbeeck, ${ }^{2}$ G. Van Tendeloo, ${ }^{2}$ \\ and V. Moshnyaga ${ }^{1}$ \\ ${ }^{1}$ Erstes Physikalisches Institut, Universität Göttingen, Friedrich-Hund-Platz 1, 37077 Göttingen, Germany \\ ${ }^{2}$ EMAT, University of Antwerp, Groenenborgerlaan 171, 2020 Antwerp, Belgium
}

(Received 8 September 2014; accepted 15 December 2014; published online 24 December 2014)

\begin{abstract}
We report an atomic layer epitaxial growth of Ruddlesden-Popper (RP) thin films of $\mathrm{SrO}\left(\mathrm{SrTiO}_{3}\right)_{\mathrm{n}}$ $(\mathrm{n}=\infty, 2,3,4)$ by means of metalorganic aerosol deposition (MAD). The films are grown on $\mathrm{SrTiO}_{3}(001)$ substrates by means of a sequential deposition of $\mathrm{Sr}-\mathrm{O} / \mathrm{Ti}-\mathrm{O}_{2}$ atomic monolayers, monitored in-situ by optical ellipsometry. X-ray diffraction and transmission electron microscopy (TEM) reveal the RP structure with $\mathrm{n}=2-4$ in accordance with the growth recipe. RP defects, observed by TEM in a good correlation with the in-situ ellipsometry, mainly result from the excess of SrO. Being maximal at the film/substrate interface, the $\mathrm{SrO}$ excess rapidly decreases and saturates after 5-6 repetitions of the $\mathrm{SrO}\left(\mathrm{SrTiO}_{3}\right)_{4}$ block at the level of $2.4 \%$. This identifies the $\mathrm{SrTiO}_{3}$ substrate surface as a source of RP defects under oxidizing conditions within MAD. Advantages and limitations of MAD as a solution-based and vacuum-free chemical deposition route were discussed in comparison with molecular beam epitaxy. ( 2014 AIP Publishing LLC.
\end{abstract}

[http://dx.doi.org/10.1063/1.4905055]

The importance of functional oxides with layered structure is evidenced by prominent examples of High- $\mathrm{T}_{\mathrm{C}}$ cuprate superconductors, ${ }^{1}$ fatigue-free $\mathrm{SrBi}_{2} \mathrm{Ta}_{2} \mathrm{O}_{9}$ ferroelectrics, ${ }^{2}$ and $\mathrm{K}_{4} \mathrm{Nb}_{6} \mathrm{O}_{17}$ photocatalysts. ${ }^{3}$ Ruddlesden-Popper ${ }^{4}$ (RP) layered crystal heterostructures with general formula $\mathrm{SrO}\left(\mathrm{SrTiO}_{3}\right)_{\mathrm{n}}$ represent an example of an intrinsic atomic layer control occurring by means of the insertion of an additional rock-salt ( $\mathrm{Sr}-\mathrm{O})$ layer in between a specific number of perovskite $\mathrm{SrTiO}_{3}$ (STO) unit cells. Conventional solid-state reaction techniques fail to grow such single phase RP samples for intermediate $n$-values, $3<\mathrm{n}<\infty$; these samples typically consist of a mixture of RP structures with different "n." RP films with $n=1-6$ can be prepared by molecular beam epitaxy ${ }^{6-8}$ (MBE). Here, we report on the preparation of RP-STO films with $n=2-4$, using atomic layer epitaxy (ALE) of $\mathrm{Sr}-\mathrm{O} / \mathrm{Ti}-\mathrm{O}_{2}$ layers realized within a metalorganic aerosol deposition (MAD) technique, equipped with optical ellipsometry for the in-situ monitoring of the ALE growth.

In Fig. 1, we present a functional scheme of our MAD installation, used to grow the RP-STO films. Being in line with the basic principle, ${ }^{9,10}$ i.e., deposition of an oxide film as a result of a heterogeneous pyrolysis reaction at the surface of a heated substrate, the apparatus contains the following important features: (a) the elaborated pneumatic nozzle ${ }^{11}$ with up to 6 liquid channels allows one to feed the nozzle with different precursor solutions; (b) by utilizing special liquid pumps, the deposition accuracy up to $\sim 10^{-4}$ u.c. of the grown layer and a wide range of deposition rates, e.g., from 0.06 up to $9.5 \mathrm{ML} / \mathrm{s}$, were achieved; (c) the film growth was monitored by optical ellipsometry, a practical alternative to RHEED, considering the vacuum-free character of MAD. The use of optical ellipsometry to control the layer thickness by monolayer oscillations is well documented for metalorganic vapor phase epitaxy of semiconducting $\mathrm{A}^{\mathrm{III}} \mathrm{B}^{\mathrm{V}}$ films. ${ }^{12,13}$ However, an in-situ optical control at the sub-monolayer level, i.e., $\mathrm{A}-\mathrm{O} / \mathrm{B}-\mathrm{O}_{2}$ atomic layering, of the perovskite structure, was not reported up to now.

The precursors $\mathrm{Sr}$ (acetylacetonate $)_{2}\left(\mathrm{P}_{\mathrm{Sr}}\right)$ and $\mathrm{Ti}$ (isoprop $)_{2}$ (tetramethylheptanedionate $)_{2}\left(\mathrm{P}_{\mathrm{Ti}}\right)$ were dissolved in dimethylformamide (DMFA) to a concentration of $0.02 \mathrm{~mol} / \mathrm{l}$. To prepare an STO film by conventional MAD, a mixed solution of Sr- and Ti-precursors, taken with a molar ratio $\mathrm{P}_{\mathrm{Sr}} /$ $\mathrm{P}_{\mathrm{Ti}}=0.75-0.83$, was sprayed using compressed air onto a $\mathrm{TiO}_{2}$-terminated $\mathrm{STO}(001)$ substrate, ${ }^{14}$ heated to $\mathrm{T}_{\text {sub }}$ $=930^{\circ} \mathrm{C}$. To grow the RP STO films, the pulses with the duration, $\tau=1-2 \mathrm{~s}$, of well-defined amounts of $\mathrm{Sr}-$ and $\mathrm{Ti}-$ precursor solutions were sequentially injected into the MAD chamber according to the RP design recipe. In between the pulses a delay time, $\delta \mathrm{t}=2-7 \mathrm{~s}$, was applied in order to pump out the remaining aerosol. To grow $\mathrm{Sr}_{n+1} \mathrm{Ti}_{n} \mathrm{O}_{3 n+1}$ films, the following sequence of precursor pulses was applied: $\left(\mathrm{P}_{\mathrm{Sr}}\left(\mathrm{P}_{\mathrm{Sr}}\right)\right.$ $\left.\left.\mathrm{P}_{\mathrm{Ti}}\right)_{\mathrm{n}}\right)_{\mathrm{N}}$. This sequence was repeated several times, $\mathrm{N}=14$ $(\mathrm{n}=4), 15(\mathrm{n}=3)$, and $19(\mathrm{n}=2)$, to obtain RP films with a total thickness, $\mathrm{d}=20-25 \mathrm{~nm}$.

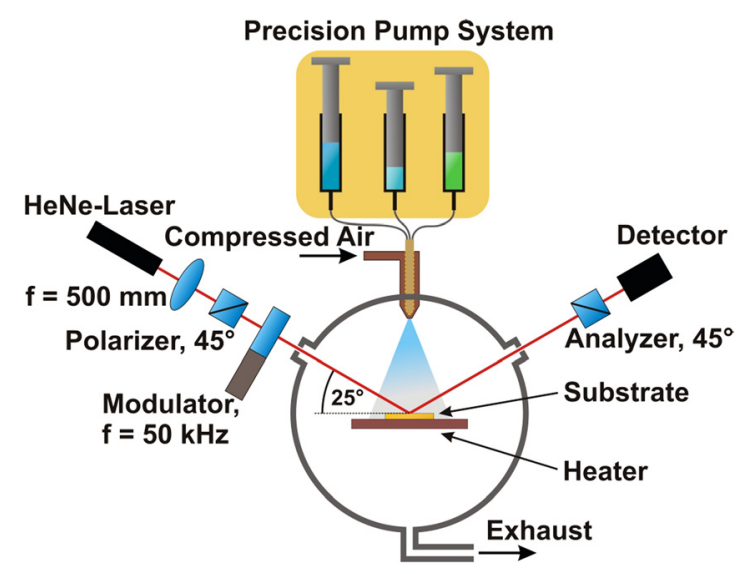

FIG. 1. Functional scheme of a MAD technique with in-situ growth control using optical ellipsometry. 
We used an optical ellipsometry setup (see Fig. 1) of the polarizer-modulator-sample-analyzer (PMSA) type ${ }^{15,16}$ with an incident $\mathrm{He}-\mathrm{Ne}$ laser beam aligned at the Brewster's angle, $\alpha \sim 65^{\circ}$, for STO. The angle of polarizer and analyzer was set to $45^{\circ}$ and the light polarization was modulated at $\omega=50 \mathrm{kHz}$ by using a photoelastic modulator (PEM). The light intensities at the fundamental, $\mathrm{I}_{\omega}$, and second harmonic, $\mathrm{I}_{2 \omega}$, frequency were measured by two lock-in amplifiers: ${ }^{15}$ $\mathrm{I}_{\omega}=\mathrm{J}_{1}(\delta) \sin (2 \Psi) \sin \Delta$ and $\mathrm{I}_{2 \omega}=\mathrm{J}_{2}(\delta) \sin (2 \Psi) \cos \Delta$. Here, $\mathrm{J}_{1}(\delta)$ and $\mathrm{J}_{2}(\delta)$ are the first and second order Bessel-function at $\delta=139^{\circ}$, the phase shift between $\mathrm{p}$ - and s-component induced by PEM. $\Psi$ and $\Delta$ are the polarization rotation angle and phase shift, respectively, between the incident and reflected beams, related to the complex reflection coefficients, $\mathrm{r}_{\mathrm{p} / \mathrm{s}}$, via $\rho=\frac{r_{p}}{r_{s}}=\tan \psi e^{i \Delta}$.

To determine the volumes of Sr- and Ti-precursors for the growth of one monolayer of $\mathrm{Sr}-\mathrm{O}$ and $\mathrm{Ti}-\mathrm{O}_{2}$, respectively, calibration experiments were carried out with volume ratios of solutions, $\mathrm{P}_{\mathrm{Sr}} / \mathrm{P}_{\mathrm{Ti}}=0.8 ; 0.775$; and 0.75 . The measured phase shift, $\Delta(\mathrm{t})$, changes periodically in time, increasing and decreasing after $\mathrm{P}_{\mathrm{Sr}}$ and $\mathrm{P}_{\mathrm{Ti}}$ pulses, respectively. The initial value $\Delta(\mathrm{t}=0)$, determined by the $\mathrm{STO}(001)$ substrate, changes very slightly during the growth, indicating a very similar dielectric properties of the grown STO film and the STO substrate. The Sr-rich composition $\left(\mathrm{P}_{\mathrm{Sr}} / \mathrm{P}_{\mathrm{Ti}}=0.8\right)$ leads to an overall increase of the $\Delta(\mathrm{t})$ signal, whereas the Sr-poor composition $\left(\mathrm{P}_{\mathrm{Sr}} / \mathrm{P}_{\mathrm{Ti}}=0.75\right)$ yields a decrease of $\Delta$. The determined optimal ratio, $\mathrm{P}_{\mathrm{Sr}} / \mathrm{P}_{\mathrm{Ti}}=0.763$, as well as the precursor volumes required for one monolayer of $\mathrm{Ti}^{-} \mathrm{O}_{2}$, $\mathrm{V}_{1 \mathrm{ML}(\mathrm{Ti})}=11 \mu \mathrm{l}$, and $\mathrm{Sr}-\mathrm{O}, \mathrm{V}_{1 \mathrm{ML}(\mathrm{Sr})}=0.763 \times \mathrm{V}_{1 \mathrm{ML}(\mathrm{Ti})}$, were further used to grow RP STO films with $n=2-4$. The calculated overall thickness of the STO $(n=\infty)$ film, $\mathrm{d}_{\text {calc }}=\mathrm{N} \times 0.3905 \mathrm{~nm}=12.1 \mathrm{~nm}$, with $\mathrm{N}=31$ periods of $\mathrm{Sr} /$ Ti-pulses fits well the thickness evaluated from $\mathrm{X}$-ray reflectometry (XRR) measurements, $\mathrm{d}_{\text {meas }}=12.9 \mathrm{~nm}$, for the sample with $\mathrm{P}_{\mathrm{Sr}} / \mathrm{P}_{\mathrm{Ti}}=0.8$. For other $\mathrm{P}_{\mathrm{Sr}} / \mathrm{P}_{\mathrm{Ti}}$ ratios, no XRR oscillations were observed because of a negligible chemical contrast. Thus, by using optical ellipsometry, we determined the stoichiometric $\mathrm{P}_{\mathrm{Sr}} / \mathrm{P}_{\mathrm{Ti}}$ ratio to grow stoichiometric $\mathrm{SrTiO}_{3}$.

The $\Delta(\mathrm{t})$ diagram in Fig. 2(a) monitors the growth of $\mathrm{N}=14$ sequences of a $\mathrm{SrO}\left(\mathrm{SrTiO}_{3}\right)_{4} \mathrm{RP}$ structure, starting with a $\mathrm{Sr}-\mathrm{O}$ layer at $\mathrm{t}_{0}=154 \mathrm{~s}$ and terminating by a $\mathrm{Ti}-\mathrm{O}_{2}$ layer at $t_{\text {end }}=1367 \mathrm{~s}$. Apparently, the ellipsometric signal oscillates along the baseline during the four circles of [Sr-O/ $\left.\mathrm{Ti}-\mathrm{O}_{2}\right]_{4}$ growth, while it shows a step-like change as soon as an additional SrO layer is added. The expected (calculated) thickness of the RP $\operatorname{STO}(n=4)$ film, $d \approx 14 \times 0.3905$ $\times(2 \mathrm{n}+1) / 2 \mathrm{~nm}=24.6 \mathrm{~nm}$, correlates well with transmission electron microscopy (TEM) data (see Fig. 4(a)). In the inset of Fig. 2(a), an AFM image of the RP STO film with $n=4$ evidences a smooth surface morphology with a root mean square roughness, RMS $=0.28 \mathrm{~nm}$. In Fig. 3(a), the $\theta-2 \theta \mathrm{X}$ ray diffraction (XRD) pattern of the RP STO film with $n=4$ exhibits peaks from the $(001)$ atomic planes indicating the out-of-plane epitaxy. From the Bragg's law with a linear dependence of $\sin \theta$ on the diffraction order, $m$ (inset of Fig. 3(a)), we calculated the c-lattice parameter of the film, $\mathrm{c}=3.59(5) \mathrm{nm}$, which fits well the literature data. ${ }^{4,7,8}$ XRD patterns for RP STO films with $\mathrm{n}=2,3$ (not shown) also reveal (00l) peaks only; the calculated c-axis parameters

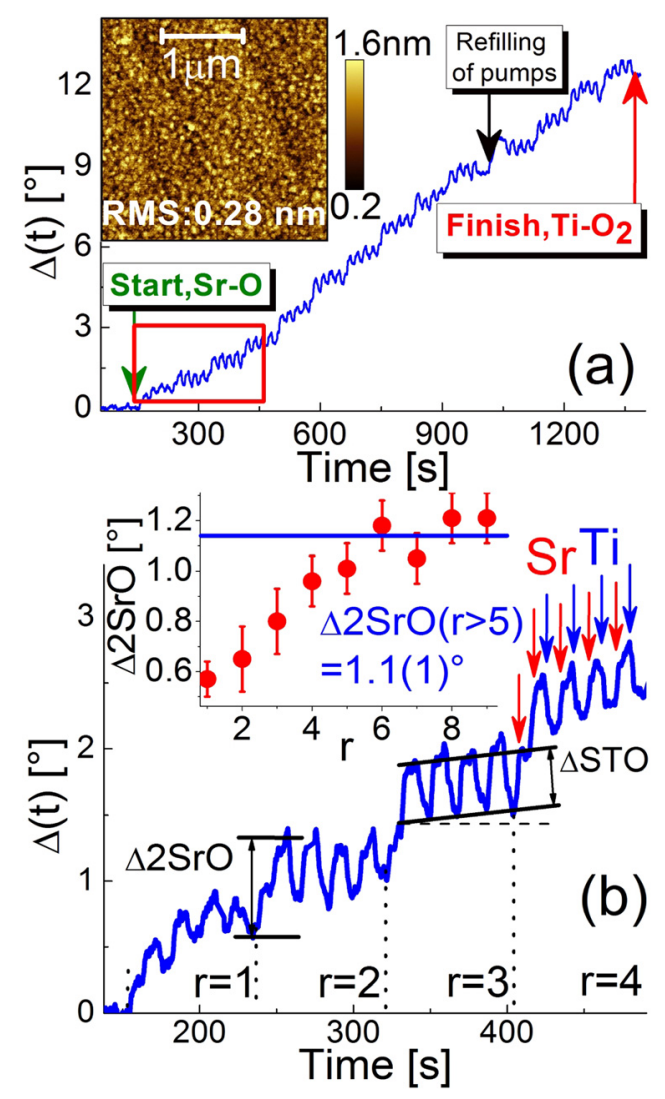

FIG. 2. (a) Heteroepitaxial growth of a Ruddlesden-Popper $\mathrm{Sr}_{5} \mathrm{Ti}_{4} \mathrm{O}_{13}$ $(\mathrm{n}=4)$ film. The inset demonstrates the AFM image. (b) Enlarged view of the first $\mathrm{r}=1-4$ repetitions of the $\mathrm{SrO}\left(\mathrm{SrTiO}_{3}\right)_{4}$ sequences (compare red box (a)). Blue and red arrows indicate the deposition of $\mathrm{TiO}_{2}$ and $\mathrm{SrO}$, respectively. $\Delta 2 \mathrm{SrO}$ is the phase shift after the deposition of two consecutive SrOlayers, and $\triangle \mathrm{STO}$ is the average oscillation amplitude during the deposition of the four STO layers. The slight upward drift during the deposition of STO indicates $\mathrm{Sr}$ excess. The inset shows the rise of $\Delta 2 \mathrm{SrO}$ for the first $\mathrm{n}$, and the average value of $\Delta 2 \mathrm{SrO}(\mathrm{r}>5)=1.1(1)^{\circ}$ for all $\mathrm{r}>5$ is also shown.

$\mathrm{c}=2.01(5) \mathrm{nm}(\mathrm{n}=2)$ and $\mathrm{c}=2.73(3) \mathrm{nm}(\mathrm{n}=3)$ agree well with the literatures. ${ }^{4,7}$,

To measure the atomic layer structure of the obtained RP films, we performed TEM analysis on the Qu-Ant-EM microscope at the University of Antwerp by means of a FEI Titan G3 instrument operating at $300 \mathrm{kV}$ acceleration voltage. TEM samples were thinned by ion milling; as preparation with focused ion beam (FIB) was found to disturb the $\mathrm{SrO}_{-} \mathrm{SrTiO}_{3}$ stacking. In Fig. 3(b), a high resolution crosssection high angle annular dark field (HAADF) scanning TEM (STEM) image of an RP STO $(n=4)$ film is presented. The extra SrO layers are clearly visible as stacking faults, shifting the bright $\mathrm{Sr}$ atomic columns in the image by a half unit cell in the horizontal direction. Such stacking corresponds apparently to the targeted sequence of layered RP structure with four STO layers separated by two SrO layers, i.e., RP STO $(n=4)$. Atomically resolved EELS mapping of $\mathrm{Sr}$ and Ti confirms this stacking sequence for a region without defects (see Fig. 3(c)). The extracted chemical maps of the corresponding Sr- $\mathrm{M}_{4 ; 5}$ and Ti- $\mathrm{L}_{2 ; 3}$ edges are presented in gray scale together with their corresponding annular dark field (ADF) survey image. For clarity, EELS line profiles across the layered RP structure are plotted in Fig. 3(c). One can clearly see the corresponding $\mathrm{SrO}$ double layers in between of 4 u.c. of $\mathrm{SrTiO}_{3}$. An overview HAADF STEM 


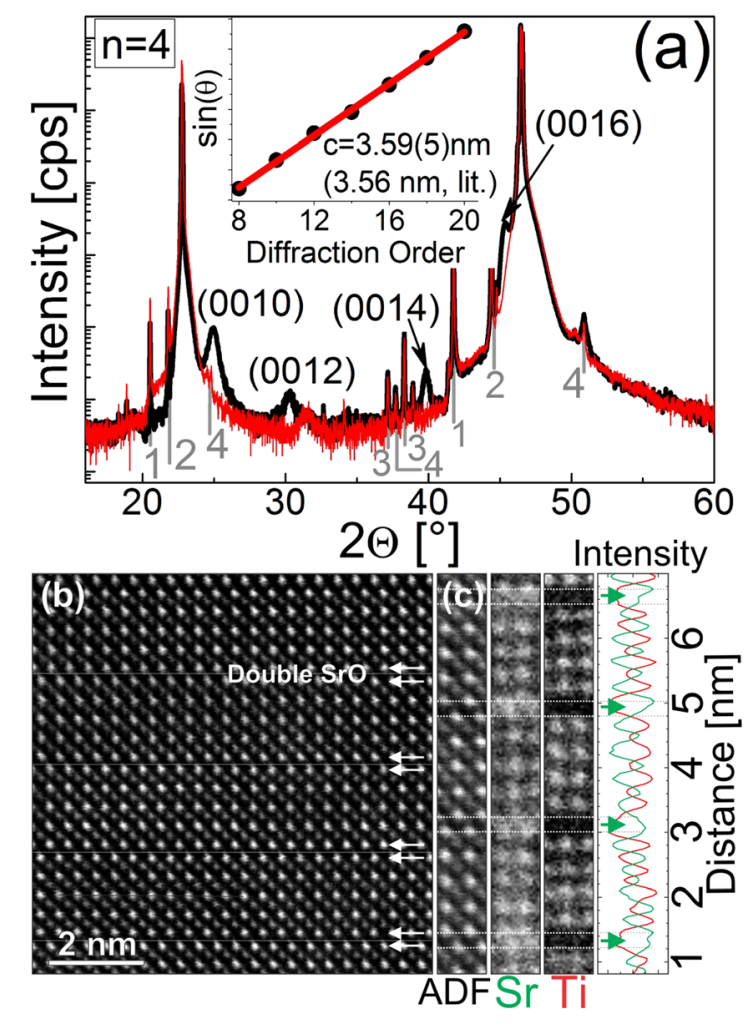

FIG. 3. (a) X-ray diffraction pattern of RP STO $(n=4)$ film with pattern of bare $\mathrm{SrTiO}_{3}(001)$ substrate (red). Spurious peaks due to contamination of the $\mathrm{Cu}-\mathrm{K} \alpha$ radiation are labeled: $1: \mathrm{Cu}-\mathrm{K} \beta, 2: \mathrm{W}-\mathrm{L} \alpha, 3: \mathrm{W}-\mathrm{L} \beta$, and $4: \mathrm{Pb}-\mathrm{L} \gamma$ (grey). Inset: Fit according to Bragg's law, $\sin \theta=(\lambda / 2 \mathrm{~d}) \times \mathrm{m}$, from which the c-lattice parameter is calculated and shown with the literature value., ${ }^{4,7,8}$ (b) High resolution HAADF-STEM image shows a film region with the desired RP $(n=4)$ structure. The double SrO layers are indicated by double white arrows. (c) EELS analysis of region with RP $(n=4)$ structure: ADF survey (left) together with elemental maps of $\mathrm{Sr}-\mathrm{M}_{4 ; 5}$ and Ti- $\mathrm{L}_{2 ; 3}$ edges presented in gray scale. In the EELS line profiles (right), the double SrO layers can be clearly identified (green arrows).

cross-section image shown in Fig. 4(a) demonstrates epitaxial growth of the RP $\operatorname{STO}(n=4) / S T O(001)$ film with the estimated total thickness, $\mathrm{d}=22 \mathrm{~nm}$. Moreover, besides the intended stacking of RP STO $(n=4)$, other periodicities with $\mathrm{n}=1-8$ appear. The extra SrO-layers shift STO-blocks along the a- and $\mathrm{b}$-axes by $\mathrm{c}_{\mathrm{STO}} / 2 \approx 0.195 \mathrm{~nm}$ and along the $\mathrm{c}$-axis by $\mathrm{c}_{\mathrm{SrO}} \approx 0.24 \mathrm{~nm}$ (lattice parameter for the extra $\mathrm{SrO}$ layers in $\mathrm{RP} \mathrm{STO}^{4,8}$ ); a similar microstructure has been observed in MBE-grown RP STO films. ${ }^{4,8}$ Vertically running SrO-layers accompany the inserted horizontal SrO-layers to balance these shifts. Due to the difference between $\mathrm{c}_{\mathrm{STO}} / 2$ and $\mathrm{c}_{\mathrm{SrO}}$ the networks of vertical and horizontal SrO-layers impose strain on the STO-blocks. Fig. 4(b) shows a sketch of the whole TEM-area in terms of STO blocks with different " $n$ " separated by $\mathrm{SrO}$ intergrowths marked by horizontal and vertical lines. The estimated amount of RP STO $(n=4)$ for the whole TEM-area, $\mathrm{A}_{\text {TEM }} \approx 49 \times 22 \mathrm{~nm}^{2}$, in Fig. 4 (a) consists of $60 \%$. Remarkably, the defects are not distributed homogeneously along the growth direction, but they rather concentrate close to the substrate within the first 5 layers. As a result in the last 5 layers in Fig. 4 the amount of the desired RP STO $(n=4)$ phase consists of $85 \%$. Quantitatively, the average SrO-excess for the first half of the film (see Fig. 4(b)) is given by 3.1 monolayers of $\mathrm{SrO}$, corresponding to a $\mathrm{Sr} / \mathrm{Ti}$ non-stoichiometry of $10.3 \%$. For the upper 5 layers,
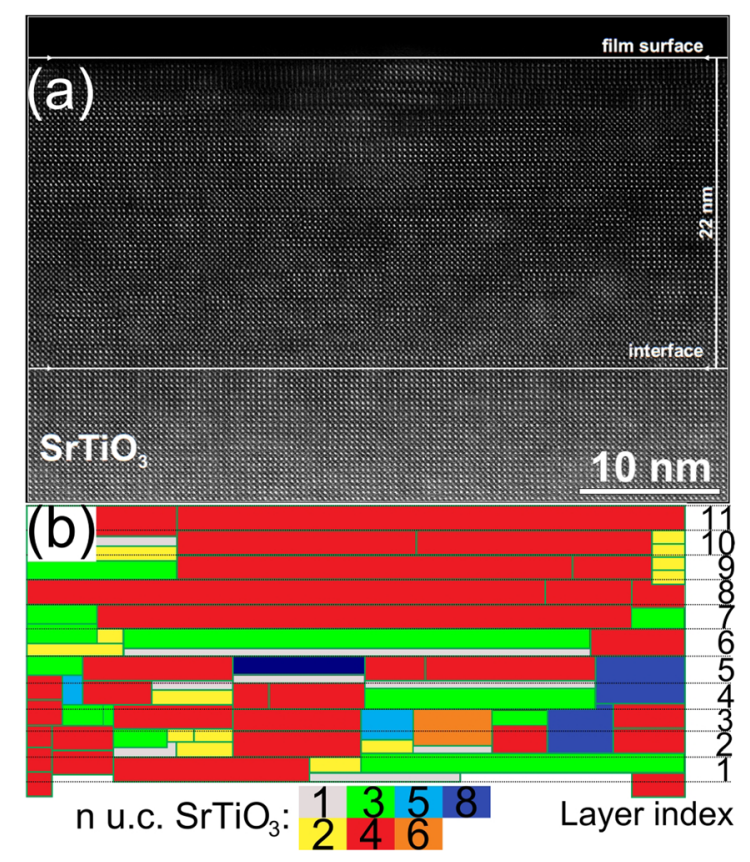

FIG. 4. (a) An overview HAADF STEM image with low resolution demonstrates epitaxial growth on $\mathrm{STO}(001)$ substrate. (b) Color coded representation of the different $\mathrm{SrTiO}_{3}$ blocks for the image shown in (a) with a thickness of $\mathrm{n}$ u.c. (see color table at the bottom) separated by vertical and horizontal SrO-layers.

the non-stoichiometry is strongly reduced down to 0.6 monolayers $(2.4 \%)$.

The important starting point to discuss the results is that we can rule out the formation of RP STO films by a solidstate reaction. Indeed, the deposition of a film from stoichiometric mixed solution for RP STO $(n=4)$ via usual MAD did not lead to an XRD pattern of any RP. Moreover, the processing conditions (annealing for several weeks at $\mathrm{T} \sim 1513 \mathrm{~K}$ ) for classical solid state routes ${ }^{5}$ differ strongly from those of MAD ALE $\left(\mathrm{T}_{\mathrm{MAD}}=1208 \mathrm{~K}, \mathrm{t}_{\mathrm{MAD}}=3600 \mathrm{~s}\right)$. With the self-diffusion coefficient of $\mathrm{D}(1208 \mathrm{~K}) \approx 1.2$ $\times 10^{-26} \mathrm{~m}^{2} \mathrm{~s}^{-1}$ for Sr in undoped STO, ${ }^{17}$ the estimated diffusion length $\sqrt{2 D t_{M A D}} \approx 0.01 \mathrm{~nm}$ is vanishingly small within MAD ALE. Finally, an additional annealing of our RP STO samples at $\mathrm{T}_{\mathrm{MAD}}=1208 \mathrm{~K}$ for $1 \mathrm{~h}$ did not alter the XRD patterns, thus, confirming a negligible effect of bulk diffusion. However, this might not be valid for SrO surface diffusion, which seems to be responsible for the intergrowths. ${ }^{5}$ Szot and Speier ${ }^{18}$ observed the enrichment of $\mathrm{SrO}$ at or just below the STO(001) surface annealed under oxidizing conditions, which is probably driven by a high surface density of dislocations, acting as diffusion channels. This is in remarkable agreement with the enhancement of RP defects close to STO substrate (see Fig. 4). Moreover, the change of the phase shift $\Delta(\mathrm{t})$ in Fig. 2(b) after the deposition of two consecutive SrO-layers, $\Delta 2 \mathrm{SrO}$, is smaller for the first 5 layers of $\mathrm{SrO}(\mathrm{STO})_{4}$ than for the later ones. This cannot be explained by a roughening of the growing SrO-layer (a roughened layer would have a smaller refractive index within an effective medium approximation ${ }^{15}$ ), but rather by the intermixing-induced formation of $\mathrm{SrO}$ intergrowths. The $\mathrm{TiO}_{2}$ layer deposited in the following step then reacts with these intergrowths to form STO with SrO-termination. As a result, one can see a smaller amplitude of $\Delta(\mathrm{t})$ oscillations 
for the first repetition of the $\mathrm{SrO}\left(\mathrm{SrTiO}_{3}\right)_{4}$ sequence $(\mathrm{r}=1$, Fig. 2(b)), i.e., the amplitude $\Delta \mathrm{STO}$ is getting progressively smaller for the four consecutive STO layers. This is also an indication for the aforementioned processes, where the termination does not change clearly from $\mathrm{TiO}_{2}$ to $\mathrm{SrO}$ but stays mainly SrO.

As the initially deposited extra layers of $\mathrm{SrO}$ have the tendency to move to the surface of the film, the substrate/film interface cannot not be sharply defined at the scale of one atomic layer (see Figs. 4(a) and 4(b)), and the film thickness might deviate slightly from the growth recipe. The reduced $\mathrm{SrO}$ non-stoichiometry in the layers at the top of the film shows that they become immobile in the later stages of the film growth. Furthermore, $\Delta 2 \mathrm{SrO}$ almost saturates after 5 repetitions (see Fig. 2(b)), signaling that the deposited double layers of $\mathrm{SrO}$ do not diffuse into the substrate for $\mathrm{r}>5$. Thus, the initial mobility of the SrO-layers seems to be related to the properties of the original substrate. After the growth of the first five repetitions of the $\mathrm{SrO}\left(\mathrm{SrTiO}_{3}\right)_{4}$ sequence, the defects of the substrate are healed and the extra SrO-layers are mostly trapped in the first half of the film. All these indicate that the growth of a $\mathrm{SrTiO}_{3}$ buffer layer with a thickness, $\mathrm{d}=25$ u.c. $\sim 10 \mathrm{~nm}$, probably will prevent the formation of the wrong SrO-STO stacking sequences. The remaining defect level of RP structure due to $\mathrm{Sr}$ excess is then intrinsically determined by the error of the $\mathrm{Sr}$ and $\mathrm{Ti}$ doses.

Finally, a comparison of atmospheric pressure MAD ALE with MBE technique with respect to the growth of RP STO films could be done. The second half of the RP STO film (Fig. 4) with $85 \%$ amount of desired phase $(n=4)$ is already not far away from $94 \%$ attained by MBE. ${ }^{8}$ Contrary to the films presented here, there are no reports about disturbed region with RP-type defects close to the surface of STObuffered STO substrates for MBE-grown films. ${ }^{6-8}$ This might be caused by different deposition conditions within MBE, i.e., partial $\mathrm{O}_{2}$ pressure during deposition $\mathrm{pO}_{2} \sim 2$ $\times 10^{-7}$ Torr and $\mathrm{T}=750^{\circ} \mathrm{C} .{ }^{8}$ Such reducing conditions could lead to different surface reactions than those for oxidizing conditions. ${ }^{18}$ Within the current MAD ALE technique with $\mathrm{pO}_{2} \sim 150$ Torr the available growth temperatures, $\mathrm{T} \sim 900^{\circ} \mathrm{C}$, are determined by the temperature necessary for the epitaxial growth of the film and by the pyrolysis reaction of the specific precursor.

To find the right Sr/Ti ratio for the deposition of stoichiometric STO, we used optical ellipsometry. The optimal ratio is accomplished when the phase shift $\Delta$ after the deposition of $\mathrm{SrO}$ and $\mathrm{TiO}_{2}$ is the same as that for the bare substrate. The accuracy of this method can be estimated to $1.5 \%$. This method and accuracy are similar to the applied RHEED technique by Haeni et al. ${ }^{19}$ to calibrate fluxes of $\mathrm{SrO}$ and $\mathrm{TiO}_{2}$ within MBE with a precision of $1 \%$. Due to the fact that the films are derived from chemical solutions, the composition of the grown films can be changed with a similar way as for the MBE. So phase diagrams of complex oxides can be studied in detail as has recently been shown for $\left(\mathrm{La}_{1-\mathrm{y}} \mathrm{Pr}_{\mathrm{y}}\right)_{0.67} \mathrm{Ca}_{0.33} \mathrm{MnO}_{3}{ }^{20}$ For the MAD ALE technique, operating at atmospheric pressure, surface contamination on the atomic scale is prevented by the relatively high substrate temperature which leads to the removal of adsorbed $\mathrm{H}_{2} \mathrm{O}$ and/or $\mathrm{CO}_{2}{ }^{21}$
In summary, the deposition of Ruddlesden Popper type $\mathrm{SrO}\left(\mathrm{SrTiO}_{3}\right)_{\mathrm{n}}(\mathrm{n}=\infty, 2,3,4)$ by a vacuum-free chemical solution-based deposition technique, MAD, is reported. The defects observed by TEM are mainly given by SrO intergrowths, the density of which decreases with increasing the film thickness and saturates after $\sim 10 \mathrm{~nm}$ at the level of $2.4 \%$ of Sr excess. We conclude that STO substrate surface enhances the mobility of the SrO layers. We expect the future improvements, tailored to the MAD technique, including surface treatments or buffer layer, will further reduce defect concentrations in RP thin films.

Financial support by the Seventh Framework Programme (Grant No. 246102 IFOX) of the EU is acknowledged. J.V. acknowledges funding from the European Research Council under the 7th Framework Program (FP7), ERC starting Grant No. 278510 VORTEX. J.V. and G.V.T acknowledge funding from the European Research Council under the 7th Framework Program (FP7), ERC Grant No. 246791COUNTATOMS. The titan microscope was partly funded by the Hercules fund from the Flemish Government. The authors acknowledge financial support from the European Union under the Seventh Framework Program under a contract for an Integrated Infrastructure Initiative, Reference No. 312483ESTEEM2.

${ }^{1}$ S. N. Putilin, E. V. Antipov, O. Chmaissem, and M. Marezio, Nature 362, 226 (1993).

${ }^{2}$ C. de Araujo, D. Cuchiaro, L. Mcmillan, M. Scott, and J. Scott, Nature 374, 627 (1994)

${ }^{3}$ A. Kudo, A. Tanaka, K. Domen, K. Maruya, K. Aika, and T. Onishi, J. Catal. 111, 67 (1988).

${ }^{4}$ S. Ruddlesden and P. Popper, Acta Crystallogr. 10, 538 (1957); 11, 54 (1958). ${ }^{5}$ R. J. D. Tilley, J. Solid State Chem. 21, 293 (1977).

${ }^{6}$ J. H. Haeni, C. D. Theis, D. G. Schlom, W. Tian, X. Q. Pan, H. Chang, I. Takeuchi, and X.-D. Xiang, Appl. Phys. Lett. 78, 3292 (2001).

${ }^{7}$ D. G. Schlom, J. H. Haeni, J. Lettieri, C. D. Theis, W. Tian, J. C. Jiang, and X. Q. Pan, Mater. Sci. Eng., B 87, 282 (2001).

${ }^{8}$ W. Tian, X. Q. Pan, J. H. Haeni, and D. G. Schlom, J. Mater. Res. 16, 2013 (2001).

${ }^{9}$ I. V. Khoroshun, E. V. Karyaev, V. T. Moshnyaga, G. A. Klosse, M. A. Krachun, V. M. Zakosarenko, and V. Yu Davydov, Supercond. Sci. Technol. 3, 493 (1990).

${ }^{10}$ V. Moshnyaga, I. Khoroshun, A. Sidorenko, P. Petrenko, A. Weidinger, M. Zeitler, B. Rauschenbach, R. Tidecks, and K. Samwer, Appl. Phys. Lett. 74, 2842 (1999).

${ }^{11}$ K. Samwer, A. Belenchuk, O. Sahpoval, and V. Moshnyaga, patent No. 10 2007055936 (2007).

${ }^{12}$ J.-T. Zettler, T. Wethkamp, M. Zorn, M. Pristovsek, C. Meyne, K. Ploska, and W. Richter, Appl. Phys. Lett. 67, 3783 (1995).

${ }^{13}$ J.-S. Lee and Y. Masumoto, J. Cryst. Growth 221, 111 (2000).

${ }^{14}$ M. Kawasaki, K. Takahashi, T. Maeda, R. Tsuchiya, M. Shinohada, O. Ishiyama, T. Yonezawa, M. Yoshimoto, and H. Koinuma, Science 266, 1540 (1994).

${ }^{15}$ H. Fujiwara, Spectroscopic Ellipsometry-Principles and Applications (John Wiley \& Sons, Ltd., 2007).

${ }^{16}$ S. N. Jasperson and S. E. Schnatterly, Rev. Sci. Instrum. 40, 761 (1969).

${ }^{17}$ K. Gömann, G. Borchardt, M. Schulz, A. Gömann, W. Maus-Friedrichs, B. Lesage, O. Kaitasov, S. Hoffmann-Eifert, and T. Schneller, Phys. Chem. Chem. Phys. 7, 2053 (2005).

${ }^{18}$ K. Szot and W. Speier, Phys. Rev. B 60, 5909 (1999).

${ }^{19}$ J. H. Haeni, C. D. Theis, and D. G. Schlom, J. Electroceram. 4, 385 (2000).

${ }^{20}$ S. Hühn, M. Jungbauer, M. Michelmann, F. Massel, F. Koeth, C. Ballani, and V. Moshnyaga, J. Appl. Phys. 113, 17D701 (2013).

${ }^{21}$ J. D. Baniecki, M. Ishii, K. Kurihara, K. Yamanaka, T. Yano, K. Shinozaki, T. Imada, K. Nozaki, and N. Kin, Phys. Rev. B 78, 195415 (2008). 